(C) [2009] IEEE. Reprinted, with permission, from [Zhen Lu, Anthony Kadi, Tim Aubrey, DA Hybrid CME-LS Channel Estimation Approach for Broadband MIMO-OFDM Systems, 2009, 9th International Symposium on Communication and Information Technology 2009, 2009]. This material is posted here with permission of the IEEE. Such ermission of the IEEE does not in any way imply IEEE endorsement of any of the University of Technology, Sydney's products or services. Internal or personal use of this material is permitted. However, permission to reprint/republish this material for advertising or promotional purposes or for creating new collective works for resale or redistribution must be obtained from the IEEE by writing to pubs-permissions@ieee.org. By choosing to view this document, you agree to all provisions of the copyright laws protecting it 


\title{
A Hybrid CME-LS Channel Estimation Approach for Broadband MIMO-OFDM Systems
}

\author{
Zhen Lu, Anthony Kadi, Tim Aubrey \\ School of Computing and Communications \\ University of Technology, Sydney, Australia \\ Tel: +61-2-95147946 \\ E-mail: zlu@eng.uts.edu.au, akadi@eng.uts.edu.au,timaa@eng.uts.edu.au
}

\begin{abstract}
A channel estimation approach yielding both channel gains and the number of multi-paths is presented. The algorithm is based on a variation of conditional model order estimation (CME), and is proposed for MIMO-OFDM systems. The CME based estimator is shown to be viable in both fixed and mobile broadband MIMO-OFDM wireless applications, and takes advantage of determining the nonzero taps as well. The performance of the approach is compared to the popular minimum description length (MDL) algorithm for estimation of the number of channel paths, by means of simulation in the context of a $2 \times 2$ MIMO-OFDM transceiver system. Results indicate that the new algorithm is superior in channel order estimation to the MDL algorithm in MMO-OFDM system over a noisy frequency selective fading channel.
\end{abstract}

\section{INTRODUCTION}

In wireless environments, signals bounce off many obstacles such as mountains, buildings, trees, etc. as they propagate between transmitters and receivers. The resultant signal at the receive antenna is, therefore, often the sum of the attenuated transmitted signal and one or more delayed versions of the transmitted signal. This received signal often suffers from intersymbol interference which degrades the signal quality to a certain extent. However, MIMO-OFDM systems are designed to take advantage of the multi-path properties in wireless communications and are capable of improving transmission rate, range and reliability simultaneously. MIMO-OFDM systems have shown great promise in a range of wireless applications and have been adopted in several wireless standards such as IEEE 802.11n, IEEE 802.16e. Such systems are also potential candidates for the fourth-generation $(4 \mathrm{G})$ systems [1].

In a MIMO-OFDM system, channel state information (CSI) can be used at the receiver to perform space-time decoding or diversity combining. Various model-based channel estimation approaches have been proposed for single antenna OFDM systems, founded on three main algorithms: maximum likelihood (ML) estimation; least squares (LS) estimation; and minimum mean squared error (MMSE) estimation [2]. In addition, channel estimation approaches can be further categorized as: pilot-assisted [3] - [5] and blind [6] - [8]. Blind techniques increase the effective throughout but either degrade system performance or lower convergence rate. In contrast, the pilot based method consumes more bandwidth, yet are less complex and improves system performance significantly [9].

In most existing estimation schemes for MIMO-OFDM there is an assumption that the number of channel taps (i.e. number of propagation paths) is known. In many practical wireless applications in which the propagation environment is both complex and time-variant, this assumption is invalid. Recently, some attention has been directed to estimating the number of multi-path taps. For instance, Yang et. al. described an algorithm for this purpose [10], in which minimum description length (MDL) was first applied in OFDM. Wang et. al. documented a probabilistic data association (PDA) based time of arrival (TOA) estimation approach for a MIMO-OFDM system, which again employed MDL to detect the number of channel paths [11]. Whilst improving performance in channel model estimation, each of these hybrid approaches is relatively costly in terms of computational complexity.

In this paper, a pilot-assisted, CME based channel estimation is proposed for broadband MIMO-OFDM system with the purpose of estimating both the number of paths and channel gains in frequency selective fading channels. Given the aim of low computational complexity, least squares (LS) is selected for use with the modified CME. Moreover, the proposed CME is capable of determining the significant (nonzero) multi-channel taps, and thus has advantages in reducing the complexity as well as improving the efficiency of MIMO system. The algorithm is then verified in both fixed and mobile wireless systems. The rest of this paper is organized as follows: Section II describes a MIMO-OFDM system model. The proposed scheme is illustrated in section III. In section IV, simulations and numerical results are presented, and the comparison between the CME and MDL based approach is made. Finally, possible improvements and further development are outlined in the conclusion

\section{A SPACE-TIME CODED MIMO-OFDM MODEL}

A space-time coded MIMO-OFDM system is depicted Fig. 1. The system consists of $N t$ transmit antennas and $N r$ receive antennas. Orthogonal space-time coding (OSTBC) is employed for it can transform the MIMO channel into simple parallel SISO channels. At time $n$, a data block is mapped and encoded into $i$ sequences of information symbols, then pilot 


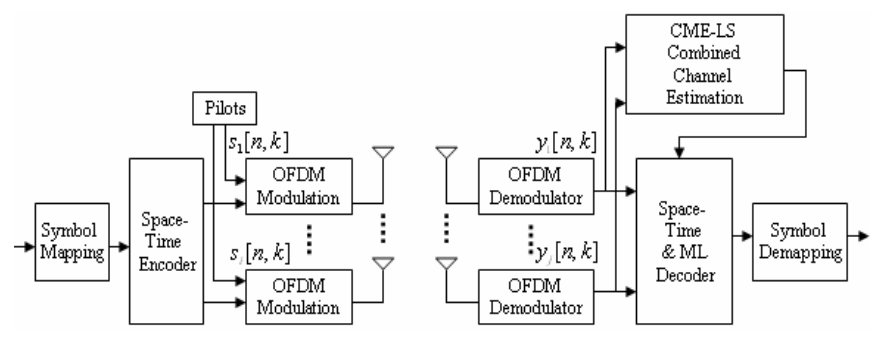

Fig. 1. MIMO-OFDM system

symbols are inserted and signal sequence $s_{i}[n, k],(k=0,1, \ldots K$; $i=1,2, \ldots N t)$ can be obtained. $K$ is the number of subchannels, and $i$ represents the $i$ th transmit antenna. Hence, the output signal of the FFT operation at the receiver is expressed as

$$
y_{j}[n, k]=\sum_{i} H_{i j}[n, k] s_{i}[n, k]+w_{j}[n, k]
$$

where $H_{i j}[n, k]$ is the channel frequency response at the $k$ th tone of the OFDM symbol transmitted from the ith transmit antenna to $j t h$ receive antenna at time $n . w_{j}[n, k]$ denotes the additive Gaussian white noise at antenna $j$ with zero mean and variance $\sigma^{2}$. The noise variance $\sigma^{2}$ is assumed unknown in the system. In order to perform decoding and diversity combining at receiver, $H_{i j}[n, k]$ should be detected. A hybrid $\mathrm{CME}$ and LS estimation approach is described in the following section.

\section{PRoposed CME-LS CHANNEL ESTIMATION}

Determination of the number of model parameters is critical in determining an optimal solution in communication systems. An effective approach, conditional model order estimator (CME), is proposed in [12]. The algorithm is based on a maximum a posterior (MAP) rule, and can be used to acquire essential parameters in terms of maximizing the conditional probability density function (PDF) of the data, without any requirements of prior knowledge. The CME rule described in [12] chooses the hypothesis that maximizes

$$
L_{X T i}(\mathbf{x})=p_{X}\left(\mathbf{x} ; \boldsymbol{\theta}_{i} \mid H_{i}\right) / p_{T i}\left(\mathbf{T} i(\mathbf{x}) ; \boldsymbol{\theta}_{i} \mid H_{i}\right) .
$$

If the noise variance is unknown, the system can be modeled as a Gaussian linear system,

$$
\mathbf{x}=\mathbf{H} \boldsymbol{\theta}+w
$$

where $\boldsymbol{H}$ is $N \times p$ matrix, $\boldsymbol{\theta}$ is $p \times 1$ matrix, w is noise vector $\left\{\mathrm{W} \sim N\left(0, \sigma^{2} \mathbf{I}\right)\right\}$. The complete derivation is illustrated in [12]. Finally, the CME rule is simplified so as to choose the model order $i$ that minimizes

$$
\begin{aligned}
& \operatorname{CME}(i)=\ln \hat{\sigma}_{i}^{2} \times(N-i-2) / 2 \\
& +\frac{1}{2} \ln \left|\mathbf{H}_{i}^{T} \mathbf{H}_{i}\right|+\ln \left\{[\pi(N-i)]^{(N-i) / 2} / \Gamma\left(\frac{N-i}{2}\right)\right\}
\end{aligned}
$$

$N$ is the number of data used to determine the model order, $i=1,2, \ldots, M, M$ is the maximum possible order, $\Gamma$ is gamma and $\Gamma(n+1)=n !,[]^{T}$ stands for transpose of a matrix, and the sufficient statistic for unknown parameters is

$$
\begin{gathered}
\hat{\sigma}_{i}^{2}=\frac{1}{N-i}\left(\mathbf{x}-\mathbf{H}_{\mathbf{i}} \hat{\boldsymbol{\theta}}_{\mathbf{i}}\right)^{T}\left(\mathbf{x}-\mathbf{H}_{\mathbf{i}} \hat{\boldsymbol{\theta}}_{\mathbf{i}}\right), \\
\hat{\boldsymbol{\theta}}=\left(\mathbf{H}^{T} \mathbf{H}\right)^{-1} \mathbf{H}^{T} \mathbf{x} .
\end{gathered}
$$

This paper proposes the use of a modified CME to estimate the number of channel paths in a MIMO-OFDM system.

\section{A. Estimation of the Number of Channel Paths}

In order to apply the CME criterion in MIMO-OFDM systems and reduce the complexity of the original CME algorithm, pilots are applied in the process of estimation. With the assistance of pilots, $\hat{\boldsymbol{\theta}}$ should be known as $(\hat{\boldsymbol{\theta}} \approx \boldsymbol{\theta})$, and thus the detection for $\hat{\boldsymbol{\theta}}$ in (6) is unnecessary. According to [12], the sufficient statistic can be defined as,

$$
\mathbf{T}\left(\mathbf{y}_{j}\right)=\left\lfloor\mathbf{s}, \hat{\sigma}_{l}^{j^{2}}\right\rfloor .
$$

Using the system mode of section II, the CME criterion is simplified and redefined as:

$$
\begin{aligned}
& p_{X}\left(\mathbf{y}_{j} ; \mathbf{s}_{i}, \sigma_{l}^{j^{2}}\right)=\left(2 \pi \sigma_{l}^{j^{2}}\right)^{\frac{-P}{2}} \\
& \times \exp \left[\frac{-1}{2 \sigma_{l}^{j^{2}}}\left(\mathbf{y}_{j}-\sum_{i=1}^{N t} \mathbf{H}_{l}^{i j} \mathbf{s}_{i}\right)^{T}\left(\mathbf{y}_{j}-\sum_{i=1}^{N t} \mathbf{H}_{l}^{i j} \mathbf{s}_{i}\right)\right]
\end{aligned}
$$

$$
\begin{aligned}
& p_{X}\left(\mathbf{T}\left(\mathbf{y}_{j}\right) ; \mathbf{s}_{i},{\sigma_{l}^{j}}^{2}\right)=p\left(\mathbf{s}_{i} ; \sigma_{l}^{j^{2}}\right) \times p\left(\hat{\sigma}_{l}^{j^{2}} ; \sigma_{l}^{j^{2}}\right) \\
& =\left[(2 \pi)^{P}\left|{\sigma_{l}^{j}}^{j^{2}}\left(\mathbf{H}_{l}^{i j} \mathbf{H}_{l}^{i j}\right)^{-1}\right|\right]^{-1 / 2} \times\left[(P-l) \hat{\sigma}_{l}^{j^{2}} / \sigma_{l}^{j^{2}}\right]^{(P-l) / 2-1} \\
& \times \exp \left[\frac{-(P-l)}{2 \sigma_{l}^{j^{2}}} \hat{\sigma}_{l}^{j^{2}}\right] \cdot\left\{(P-l) /\left[\sigma_{l}^{j^{2}} \times 2^{(P-l) / 2} \Gamma\left(\frac{P-l}{2}\right)\right]\right\} \\
& \left(\hat{\sigma}_{l}^{j}\right)^{2}=\frac{1}{P-l}\left(\mathbf{y}_{j}-\sum_{i=1}^{N t} \mathbf{H}_{l}^{i j} \mathbf{s}_{i}\right)^{T}\left(\mathbf{y}_{j}-\sum_{i=1}^{N t} \mathbf{H}_{l}^{i j} \mathbf{s}_{i}\right)
\end{aligned}
$$

Therefore, from (2), (7), (8), (9),

$$
\begin{aligned}
& L_{X T i}(y)=p_{X}\left(\mathbf{y} ; \mathbf{s}_{i}, \sigma_{l}^{j}\right) / p_{T i}\left(\mathbf{T} i(\mathbf{y}) ; \mathbf{s}_{i}, \sigma_{l}^{j}\right) \\
& =\Gamma\left(\frac{P-l}{2}\right) /\left\{[\pi(P-l)]^{(P-l) / 2}\left|\mathbf{H}_{l}^{i j} \mathbf{H}_{l}^{i j}\right|^{1 / 2}\left(\hat{\sigma}_{l}^{j^{2}}\right)^{(P-l) / 2-1}\right\}
\end{aligned}
$$

Equation (11) can be finally expressed as

$$
\begin{aligned}
& \operatorname{CME}_{\mathrm{ij}}(l)=[(P-l-2) / 2] \times \ln \left(\hat{\sigma}_{l}^{j}\right)^{2}+ \\
& \frac{1}{2} \ln \left|\left(\mathbf{H}_{l}^{i j}\right)^{T} \mathbf{H}_{l}^{i j}\right|+\ln \left\{[\pi(P-l)]^{(P-l) / 2} / \Gamma\left(\frac{P-l}{2}\right)\right\}
\end{aligned}
$$

where $\left(\hat{\sigma}_{l}^{j}\right)^{2}$ is given in (9), $\mathbf{H}_{l}^{i j}$ is the estimated channel frequency response between ith transmitter antenna and jth 
receiver antenna obtained by LS estimation, $\boldsymbol{s}_{i}$ are the pilots delivered from ith antenna, $P$ is the total number of pilots and $l=1,2, \ldots, L$, where $L$ stands for maximum possible number of channel paths. Therefore, the number of channel paths can be finally detected by minimizing CME,

$$
\hat{l}=\arg \min _{l}\left\{C M E_{i j}\left(\mathbf{y}_{j}, l\right)\right\} .
$$

In order to acquire the number of channel taps, the channel frequency response is required. Here a least squares (LS) approach is used for this purpose due to its low computational complexity. However the proposed CME can be combined with other channel detection methods such as minimum mean square error (MMSE) estimation, which are capable of achieving better performance.

\section{B. Pilot Assisted LS Estimator}

The frequency selective channel is assumed to be Rayleighfading, with frequency response: $\mathbf{H}_{i j}^{p}$ and impulse response: $h_{i j}[l], 1=0,1, \ldots, L-1$, corresponding to the channel between ith transmit-antenna and $j$ th receive-antenna, and $L$ is the number of channel taps. The received pilot symbols at the $j$ th receive antenna are

$$
\mathbf{y}_{j}^{p}=\sqrt{\varepsilon_{p}} \sum_{i} \mathbf{H}_{i j}^{p} \mathbf{s}_{i}^{p}+w_{j}^{p}, p \in I_{p}
$$

where $w_{j}^{p}$ is additive white Gaussian noise (AWGN), $\varepsilon_{p}$ is the transmitted power per pilot symbol, $I_{p}$ denotes the position of pilot sub-carriers, and $P$ is the number of pilots. Using [13], for both transmitter and receiver diversity scheme, the following is defined

$$
\mathbf{G}_{i}=\left(\varepsilon_{P} \mathbf{F}_{P} D^{H}\left(\mathbf{s}_{i}^{p}\right) D\left(\mathbf{s}_{i}^{p}\right) \mathbf{F}_{p}^{H}\right)^{-1} \times\left(\sqrt{\varepsilon_{p}} D\left(\mathbf{s}_{i}^{p}\right) \mathbf{F}_{p}^{H}\right)^{H}
$$

and the LS estimate of channel impulse is given by

$$
\hat{\mathbf{h}}_{i j}=\mathbf{G}_{i} \mathbf{y}_{j}^{p}
$$

where $\mathbf{F}$ is a $L \times K$ matrix $[\mathbf{F}]_{l, k}=\exp (j 2 \pi(l-1)(k-1) / K)$, $\mathbf{f}_{i p},\left(\{i p\}_{i p=1}^{p}=I_{p}\right)$ is $p$ th column of $\mathbf{F}, \mathbf{F}_{p}=\left[\boldsymbol{f}_{l}, \ldots, \boldsymbol{f}_{i p}, \ldots, \boldsymbol{f}_{p}\right]$, and thus $\mathbf{F}=\left[\boldsymbol{f}_{l}, \ldots, \boldsymbol{f}_{n}, \ldots, \boldsymbol{f}_{K}\right] ; \mathbf{s}_{i}^{p}$ is the transmitted pilot symbols. In addition, $D(x)$ stands for a diagonal matrix with $x$ in its diagonal, and []$^{H}$ is the Hermitian transpose. Thus, the estimated channel frequency response is

$$
\hat{\mathbf{H}}_{i j}=\mathbf{f}_{n}^{H} \hat{\mathbf{h}}_{i j} .
$$

Overall, the hybrid CME-LS algorithm can be regarded as a decision feedback approach which is shown in Fig.2.

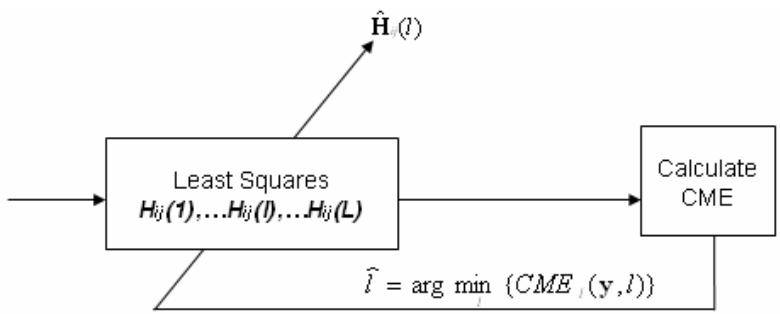

Fig. 2. Hybrid CME-LS channel estimation

\section{Simulation AND Results}

A $2 \times 2$ MIMO-OFDM system with Alamouti space-time code [14], which is the best known OSTBC, is employed to demonstrate the performance of proposed hybrid approach in multi-path Rayleigh fading channels.

\section{A. Performance Comparison for CME \& $M D L$}

The performance of $\mathrm{CME}$ and minimum description length (MDL) is compared through simulation. MDL has been employed in OFDM system in [10] and MIMO-OFDM in [11]. According to [12], the MDL for MIMO system can be expressed as,

$$
\begin{gathered}
M D L_{i j}(l)=(P / 2) \ln \left(\hat{\sigma}_{l}^{j}\right)^{2}+[(L+1) / 2] \ln P \\
\left(\hat{\sigma}_{l}^{j}\right)^{2}=\left(y_{j}-\sum_{i} \mathbf{H}_{l}^{i j} \mathbf{s}_{i}\right)^{T}\left(y_{j}-\sum_{i} \mathbf{H}_{l}^{i j} \mathbf{s}_{i}\right) / P \\
\hat{l}=\arg \min _{l}\left\{M D L_{i j}\left(\mathbf{y}_{j}, l\right)\right\}
\end{gathered}
$$

2000 OFDM symbols are employed to compare the performance of CME and MDL. Each OFDM symbol contains $K$ sub-carriers $(K=256)$ in which $P(P=8)$ pilots are utilized to detect both number of channel taps and channel gains, the cyclic prefix (CP) number is chosen as $G=K / 4=64$. With the aim of testing the proposed approach, the assumption that the maximum delay of multi-path fading channels is less than the cyclic prefix period and can thus be ignored, is made. The channel model is assumed quasi-static.

Fig. 3 indicates the comparison of probability of correct detection of channel model order between CME and MDL at different SNR when the number of channel paths $L=1,2,3$ and 4 , respectively. Given that the model order estimator is affected by noise significantly, the detection begins from $\mathrm{SNR}=10 \mathrm{~dB}$. The results show that the CME is superior to MDL in estimation of the number of channel paths. It should be noted that there is an upper limit to the quantity of taps that can be accurately estimated based on the number of pilot tones used. According to the research, in order to correctly detect $L$, the number of pilots $P$ should be: $P \geq 2 L+1$, and thus no more than 3 channel paths can be detected in the simulation above since only 8 pilot symbols are employed. Using the same simulation parameters as above and in the case of $\mathrm{SNR}=20 \mathrm{~dB}$, a probability of only around $30 \%$ is achieved for $L=4$ (See Fig.3).

Therefore, for $L \geq 4$, the 8 pilot estimator is insufficient. Increasing the number of pilots will increase the estimation 

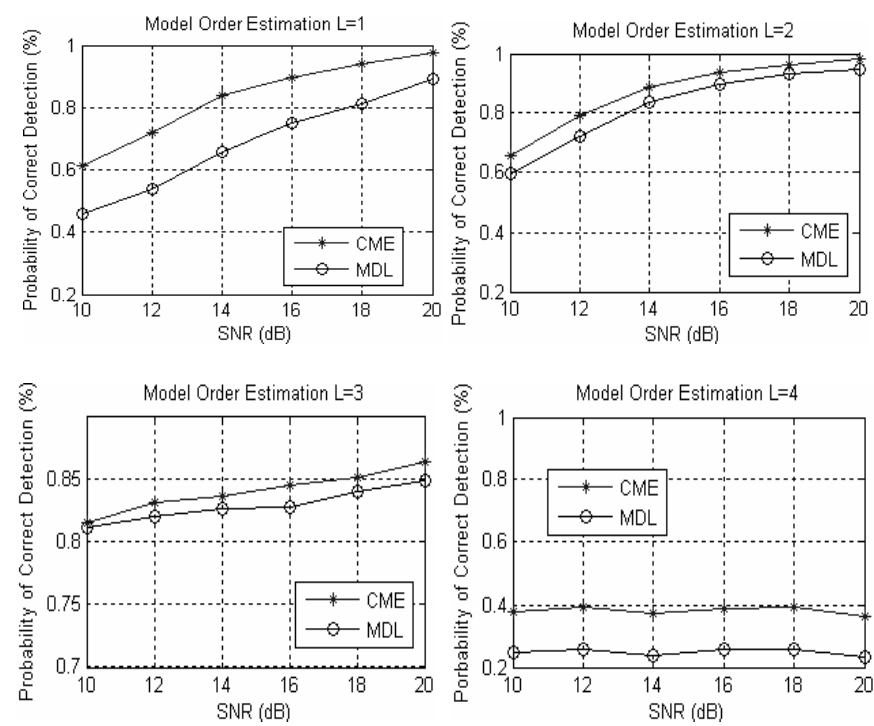

Fig.3. Comparison of probability of correct detection between CME \& MDL with 8 pilots

accuracy for more complex propagation environments, but will also reduce bandwidth efficiency. In many common propagation environments, it may be possible to assume that there are only 3 or less dominant transmission paths. Where such an assumption does not hold, more pilots should be employed, despite the consumption of additional bandwidth. For a more complex wireless environment, 16 pilot symbols are used and accuracy results are given in Fig.4. In this case, more pilots are utilized and the number of channel paths that can be correctly detected increases. A comparison of both estimators in Table I shows that CME still outperforms MDL overall.

Simulated BER performance for BPSK modulation is depicted in Fig. 5. Here LS estimation - with known number of taps $(L=4)$, CME-LS and MDL-LS estimation are compared. It can be seen that the performance of CME is quite close to LS with known $L$. At higher SNRs the CME

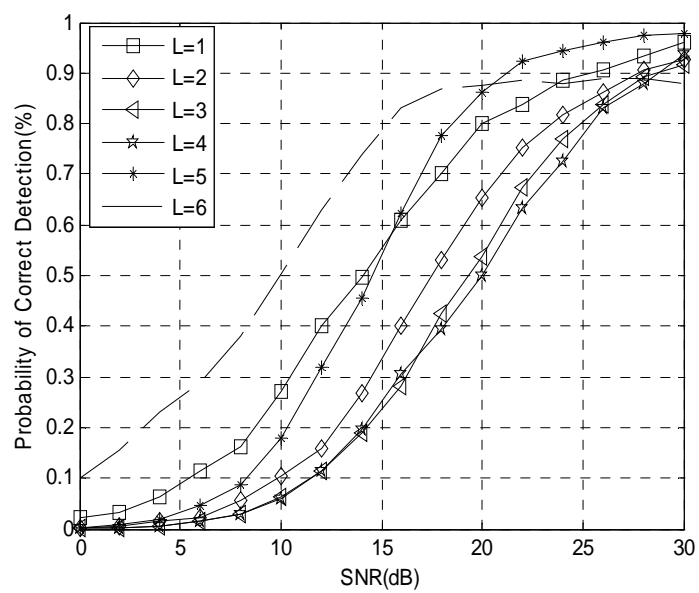

Fig.4. Probability of correct detection for CME with 16 pilots
TABLE I

COMPARISON OF CME \& MDL WITH 16 PILOTS

\begin{tabular}{|c|c|c|c|c|c|c|c|}
\hline & & \multicolumn{5}{|c|}{ Actual number of taps (L) } \\
\hline SNR=30dB & Algorithm & $\mathrm{L}=1$ & $\mathrm{~L}=2$ & $\mathrm{~L}=3$ & $\mathrm{~L}=4$ & $\mathrm{~L}=5$ & $\mathrm{~L}=6$ \\
\hline $\begin{array}{c}\text { Probability of } \\
\text { correct detection }\end{array}$ & $\mathrm{CME}$ & $96.70 \%$ & $93.45 \%$ & $91.30 \%$ & $90.50 \%$ & $98.60 \%$ & $88.40 \%$ \\
\cline { 2 - 7 } & MDL & $75.00 \%$ & $94.65 \%$ & $88.60 \%$ & $87.90 \%$ & $56.75 \%$ & $54.55 \%$ \\
\hline
\end{tabular}

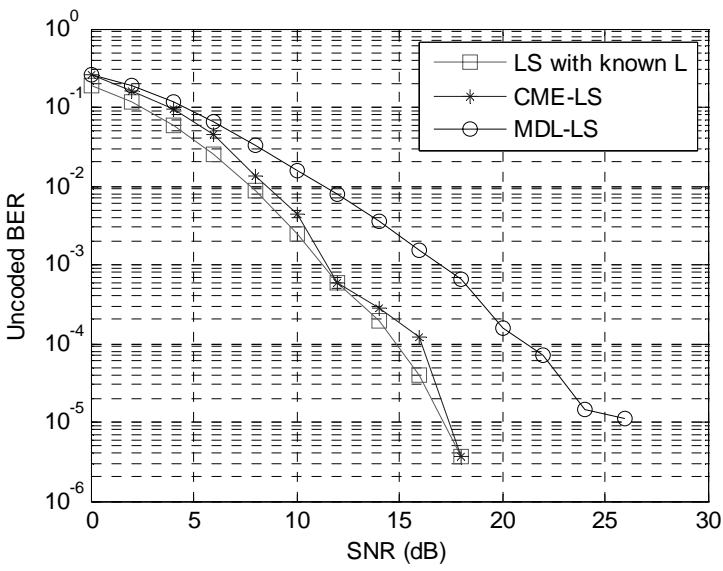

Fig.5. Comparison of BER performance of LS with known number of taps (L), CME-LS \& MDL-LS with 16 pilots

detector works quite well and achieves better performance than that of MDL-LS, demonstrating its advantage in such situations. However, by taking the computational complexity into account, MDL is better than $\mathrm{CME}$, the ratio of average CPU time requirement of MDL is only $80 \%$ to that of the proposed CME. Nevertheless, the proposed algorithm is preferred due to its significant performance gain.

\section{B. CME-LS in Nonzero Taps Estimation}

In practice, wireless multi-path channels are dominated by a relatively small number of significant paths, and detecting nonzero taps becomes a critical approach in reducing algorithm complexity and improving system efficiency. The proposed approach is also verified as an effective method for determining the number of key taps in a MIMO-OFDM system. Examples obtained from the simulation with 8 pilots are shown in Table II. 100 packets of data are utilized as the input signal, the actual number of multi-path taps is set to 3, with one of the paths having a much lower power than the others. $\quad\left(P_{h i}=\left|h_{i}\right|^{2}<2|w|^{2}, \quad|w|^{2} \quad\right.$ is noise power, $\left.i=1,2,3\right)$. According to Table II, the majority of the packets only detect two paths in spite of three taps being present in the system (e.g. $\mathrm{SNR}=20 \mathrm{~dB}, 99$ out of 100 packets determine that the

TABLE II

CME IN NON-ZERO TAPS DETECTION FOR MIMO-OFDM

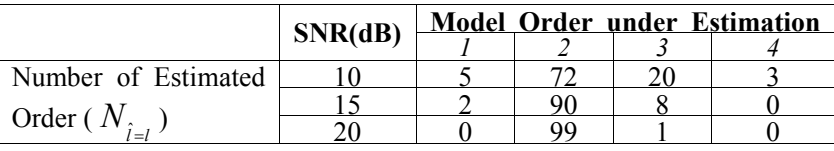


number of taps is 2). The performance of the proposed CME improves significantly as noise decreases.

\section{CME-LS for Fixed Broadband MIMO-OFDM System}

Parameters for a fixed wireless system are set according to IEEE 802.16-2004 [15], each OFDM symbol contains $K$ subcarriers $(K=256)$ in which 192 tones are used for data transmission and $P(P=8)$ pilots are utilized to detect both channel taps and channel gains, the remaining sub-carriers are employed as lower and upper guards. The cyclic prefix (CP) number is chosen as $G=K / 4=64$. The system bandwidth is $1.75 \mathrm{MHz}$, and the sampling factor is $8 / 7$. Since $256 \mathrm{sub}$ channels are used, the useful symbol time is $128 \mu$ s and CP time is $32 \mu \mathrm{s}$. The system is operating on SUI-4 channel model, which is shown in Table III

TABLE III

SUI-4 CHANNEL MODEL

\begin{tabular}{c|c|c|c}
\hline & Tap 1 & Tap2 & Tap3 \\
\hline Delay $[\mu \mathrm{s}]$ & 0 & 1.5 & 4.0 \\
\hline Power $[\mathrm{dB}]$ & 0 & -4 & -8 \\
\hline Doppler $[\mathrm{Hz}]$ & 0.20 & 0.15 & 0.25 \\
\hline
\end{tabular}

BPSK is used in the simulation. The probability of correct detection for CME-LS in fixed broadband MIMO-OFDM is shown in Fig.6. 2000 OFDM symbols are utilized in the simulation. The comparison of BER performance between CME-LS estimation and LS with known number of channel taps $L$ is depicted in Fig.7. The results confirm that CME-LS works well in estimation the number of multi channel paths in fixed Broadband wireless system.

\section{CME-LS for Mobile Broadband MIMO-OFDMA System}

The mobile system simulation is based on the IEEE 802.16e [16] standard, where MIMO-OFDMA is applied instead of MIMO-OFDM. OFDMA (Orthogonal Frequencydivision Multiple Access) uses the same techniques as OFDM, but allows for a multiple-access function. Downlink partial usage of sub-channels (DL-PUSC) is employed, and subcarriers are divided into physical clusters, each of which contains 14 adjacent subcarriers. In contrast to full usage of sub-channels (FUSC), the pilots for PUSC allocated to subchannels, with each subchannel having its own pilot arrangement [16]. For OFDMA, the physical clusters are renumbered into logical cluster, which are then divided into 6 sub-channel major groups and finally allocated to several segments of the downlink sub-frame. In a segment, a user is only able to obtain information from the clusters allocated to him, and at least one group of cluster shall be allocated. In this paper, the channel estimation is carried out per group, assume that only one major group allocated to a user in a segment, and thus we can focus on demonstration of the proposed channel estimation approach in the broadband mobile system. According to IEEE 802.16e, there are four OFDMA symbol structures with FFT sizes of 128, 512, 1024 and 2048, respectively. The OFDMA symbol parameters for simulation and the channel model "Vehicular A" are shown in Table IV and Table V, respectively.

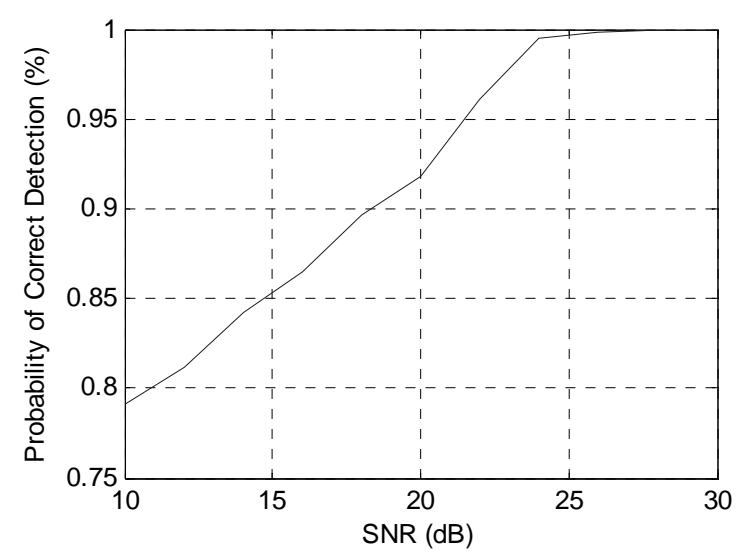

Fig.6. Probability of correct detection for CME-LS with 8 pilots

TABLE IV

SYSTEM PARAMETERS FOR MOBILE SYSTEM

\begin{tabular}{|l|c|}
\hline parameter & \\
\hline FFT size & 1024 \\
\hline Number Of used subcarriers & 841 \\
\hline Number of guard subcarriers & $92+91$ \\
\hline Cyclic prefix & $1 / 8$ \\
\hline Channel bandwidth $(\mathrm{MHz})$ & 10 \\
\hline Sampling frequency $(\mathrm{MHz})$ & 11.2 \\
\hline Subcarrier spacing $(\mathrm{KHz})$ & 10.94 \\
\hline Useful symbol time $(\mu \mathrm{s})$ & 91.4 \\
\hline CP time $(\mu \mathrm{s})$ & 11.4 \\
\hline OFDM duration $(\mu \mathrm{s})$ & 102.9 \\
\hline
\end{tabular}

TABLE V

"VeHICUlAR A" CHANNEL ENVIRONMENT

\begin{tabular}{c|c|c}
\hline Tap & Time delay $(\mu \mathrm{s})$ & Average power $(\mathrm{dB})$ \\
\hline 1 & 0 & 0 \\
\hline 2 & 0.31 & -1 \\
\hline 3 & 0.71 & -9 \\
\hline 4 & 1.09 & -10 \\
\hline 5 & 1.73 & -15 \\
\hline 6 & 2.51 & -20
\end{tabular}


For OFDMA with 1024 subcarriers, there are two kinds of groups: one contains 12 clusters and another includes 8 clusters; 2 pilots are inserted in each cluster according to the standards [16]. In the simulation, we choose to show the performance of the group with 8 clusters, and thus 16 pilot symbols can be used. QPSK modulation is utilized. The comparison of BER performance between CME-LS and LS with known number of channel paths $(L)$ is presented in Fig.8. The Doppler shift for the mobile system is set as $f d=40 \mathrm{~Hz}$, where Rayleigh fading is generated by Jake's model. The results indicated that the proposed algorithm is capable of obtaining almost the same results as that with known channel tap length in mobile broadband MIMO-OFDM system.

\section{CONCLUSION}

In this paper, the proposed pilot-assisted CME based channel estimation algorithm has been shown to be a viable approach in determining the number of channel paths, especially nonzero paths, for broadband MIMO-OFDM systems in the absence of prior knowledge of model parameters. A comparison of the performance between the modified CME and MDL has shown that the former is superior. Although the simulations have been done in relatively simple wireless environments, i.e. the number of channel taps is less than or equal to 3 , it is possible to apply the estimation scheme in more complex environments by increasing the number of pilot tones. The algorithm also is verified its capacity in both fixed and mobile MIMO-OFDM wireless system based on IEEE $802.16 \mathrm{~d} / \mathrm{e}$ standard. While the modified CME algorithm is combined with LS channel estimation, it is not restricted to this approach and can be combined with other estimation schemes. For example, a hybrid CME-MMSE algorithm could improve the system BER performance at the cost of increased computational complexity. The benefit of the CME approach to estimating the number of channel paths has been established. Further research into hybrid methods employing this approach is warranted, and hardware demonstration is in consideration.

\section{REFERENCES}

[1] G. L. Stuber, J. R. Barry, S. W. McLaughlin, Y. Li, M. A. Ingram and T. G. Pratt, "Broadband MIMO-OFDM wireless communications"', Proceedings of the IEEE, vol. 92, no. 2, pp. 271-294, 2004.

[2] J S. M. Kay, Fundamentals of Statistical Signal Processing: Estimation Theory, PTR Prentice-Hall, Englewood Cliffs, N.J, 1993.

[3] J. K. Cavers, "An analysis of pilot symbol assisted modulation for Rayleigh fading channels [mobile radio]", Vehicular Technology, IEEE Transactions on, vol. 40, no. 4, pp. 686-693, 1991.

[4] Y. Li, N. Seshadri, and S. Ariyavisitakul, "Channel estimation for OFDM systems with transmitter diversity in mobile wireless channels", Selected Areas in Communications, IEEE Journal, vol. 17, no. 3, pp. 461-471, 1999.

[5] Y. Li, "Simplified channel estimation for OFDM Systems with multiple transmit antennas", Wireless Communications, IEEE Transactions on, vol. 1, No. 1, pp67-75, 2002.

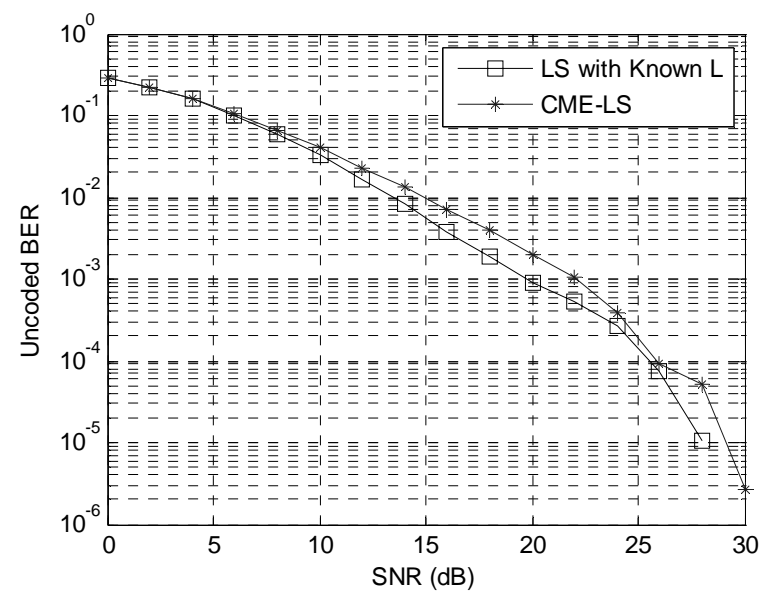

Fig.8. Comparison of BER performance between CME - LS \& LS with known number of channel taps (L) in mobile system

[6] Z. Ding, and Y. Li. Blind channel equalization and identification, Marcel Dekker, New York, 2000.

[7] M. C. Necker and G. L. Stüber, "Totally blind channel estiamtion for OFDM on fast varying monile radio channels," IEEE Trans. Wireless Commun., vol 3, no. 5, pp. 1514-1525, Sep. 2004

[8] I. Bradaric, A. P. Pertropulu and K. I. Diamantaras, "Blind MIMO FIR channel identification based second-order spectra correlations," IEEE Trans, Signal Process., vol. 51, no. 6, pp. 1668-1674, Jun. 2003

[9] M. Jiang and L.Hanzo, "Multiuser MIMO-OFDM for nextgeneration wireless systems,"Processings of the IEEE, vol. 95, no. 7, July, 2007

[10] B. Yang, K.B. Letaief, R.S. Cheng and C. Zhigang. "Channel estimation for OFDM transmission in multipath fading channels based on parametric channel modelling", Communications, IEEE Transactions on, vol. 49, no. 3, pp. 467-479, 2001

[11] J. Wang, H. Zhu and K. J. R. Liu. "A MIMO-OFDM channel estimation approach using time of arrivals", Wireless Communications, IEEE Transactions on, vol. 4, no. 3, pp. 12071213,2005

[12] S. Kay. "Conditional model order estimation", Signal Processing, IEEE Transactions on, vol. 49, no. 9, pp. 1910-1917, 2001

[13] X. Cai \& G .B. Giannakis. "Error probability minimizing pilots for OFDM with M-PSK modulation over Rayleigh-fading channels", Vehicular Technology, IEEE Transactions on, vol. 53, no. 1, pp. 146-155, 2004

[14] S. M. Alamouti. "A simple transmit diversity technique for wireless communications", Selected Areas in Communications, IEEE Journal on, vol. 16, no. 8, pp. 1451-1458, 1998.

[15] IEEE 802.16-2004, "IEEE Standard for Local and Metropolitan Area Networks - Part 16: Air Interface for Fixed Broadband Wireless Access Systems", October 2004

[16] IEEE P802.16e/D8, "Amendment for Physical and Medium Access Control Layers for Combined Fixed and Mobile Operation in Licensed Bands", May 2005. 